\title{
Dimensions and Refractive Index Estimates of Deeply Buried Optical Waveguides in Lithium Fluoride
}

\author{
Ismael Chiamenti ${ }^{1}$, Francesca Bonfigli ${ }^{2}$, Rosa Maria Montereali ${ }^{2}$ and Hypolito J. Kalinowski ${ }^{1}$ \\ 1. Federal University of Technology - Paraná, 80230-901, Curitiba, Brazil \\ 2. ENEA C. R. Frascati, Photonics Micro and Nanostructures Laboratory, UTAPRAD-MNF, 00044 \\ Frascati $(R M)$, Italy \\ E-mail address: hjkalin@utfpr.edu.br (H.J. Kalinowski)
}

\begin{abstract}
A recursive procedure is applied to the measured nearfield profiles of buried optical waveguides recorded in a lithium fluoride ( $\mathrm{LiF}$ ) crystal by femtosecond laser pulses in order to estimate the core dimensions and the refractive index increase. Albeit the waveguides transversal section geometry is quite complex it is possible to obtain the horizontal and vertical widths and the average refractive index maximum increase assuming a simplified rectangular transversal section in the simulation. The procedure is validated by comparing the simulated results with the experimental near-field profiles and the maximum refractive index values of two commercial optical fibers. Typical dimensions of $\sim(8 \times 10) \mu \mathrm{m}^{2}$ and refractive index changes of $\sim(2-10) \times 10^{-4}$ were obtained for the $\mathrm{LiF}$ waveguides at several wavelengths.
\end{abstract}

Index Terms - Refractive Index Increase, Core Dimension, Optical Waveguide, Lithium Fluoride, Femtosecond Laser.

\section{INTRODUCTION}

The dimensions and refractive index (RI) of the core are important parameters for optical waveguides characterization. These two main attributes and the wavelength behavior permit to specify the light acceptance into the waveguide (numerical aperture), the propagation condition, dispersion relation, electromagnetic profile and cutoff frequency of a supported mode, as well as the coupling coefficient to other waveguides. All of these parameters can be controlled by changing the core dimensions, the core refractive index or both [1].

The increase $(\Delta n)$ in the spatial RI profile and the waveguide core dimensions can be estimated by different techniques, such as reflectivity method [2], scattering pattern [3], surface plasmon resonance [4], prism coupling [5, 6], surface topography [7], refraction method and $\Delta n$ estimate from near field profile (NFP) $[8,9]$. An inversion of the scalar wave equation to estimate the dimensions and the RI profile of the waveguide is possible with good signal noise ratio NFP [10].

The experimental requirements associated to the mentioned techniques turn difficult their application to analyze the RI profile and core dimensions of deep buried optical waveguides recorded by high intensity pulsed laser beams. This recording technique consists of focusing a laser beam in a 
sample that is displaced perpendicular or longitudinal with respect to the laser propagation direction $[11,12]$. The focused laser spot induces modifications of the sample structure resulting in RI changes. The RI profile recorded by this technique usually does not show a regular transverse shape $[13,14]$.

Recently, the writing of fs induced waveguides in Lithium Fluoride and several of their characteristics were reported $[15,16]$, the observed transversal cross section is also not regular, as can be seen in Fig. 1, which shows fluorescence confocal laser microscopy images of the transverse plane from (a) the side face and (b to d) from the bulk of a lithium fluoride crystal, where several optical waveguides were recorded by a femtosecond pulsed laser. The visible fluorescence is due to the local formation of laser-active aggregate color-centers in the $\operatorname{LiF}$ matrix $[15,16]$. Figure 1(e) shows the NFP measured from one waveguide when using an ASE source, with central wavelength at $1550 \mathrm{~nm}$, to launch light into the waveguide.

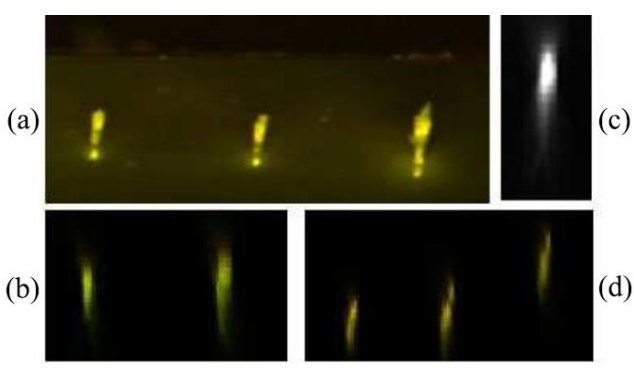

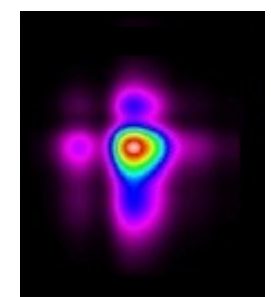

(e)

Fig. 1. Core transverse section of optically recorded color-center waveguides in a LiF crystal as seen by fluorescence confocal laser microscopy: (a) side face, (b-d) inside the crystal and (e) the NFP from waveguide \#4, @ 1550 nm,

In this manuscript we detail the recursive procedure used to estimate the average $\Delta n$ and transverse dimensions of that optical waveguides, deeply recorded by intense laser pulses in an optically transparent material such as LiF. The approach uses a non destructive technique to measure the NFP. Assuming a rectangular core with step refractive index profiles, a recursive procedure is applied to the experimental data. The approach is validated on two commercial optical fibers, comparing the obtained results with their known parameters. Subsequently the procedure is used to estimate the average $\Delta \mathrm{n}$ and the transverse dimensions of optical waveguides recorded $\sim 160 \mu \mathrm{m}$ deep from the top polished surface in a LiF crystal, at several wavelengths.

\section{MATERIALS AND METHODS}

\section{A. Numerical Modeling}

The proposed procedure uses (i) the NFP measured at a specific wavelength $(635 \mathrm{~nm}$, the measured data is treated with $1 \mathrm{~Hz}$ low-pass filter in order to reduce the numerical error in the second derivatives calculation [8]) to estimate the maximum $\Delta n$ values in the horizontal and vertical directions, $\Delta n_{\mathrm{H}}\left(x, y_{0}\right)$ and $\Delta n_{\mathrm{V}}\left(x_{0}, y\right)$, respectively. The estimation uses an inversion of the scalar wave equation obtained from [8] 


$$
\nabla_{\mathrm{T}}^{2} \psi(x, y)+\left[k_{o}^{2} n^{2}(x, y)-\beta^{2}\right] \psi(x, y)=0
$$

where $\nabla_{\mathrm{T}}^{2}$ is the Laplacian operator in the plane transversal to the propagation direction, $\psi(x, y)$ is the field function amplitude, $k_{o}$ the vacuum wave number, $n$ the refractive index of the medium and $\beta$ the propagation constant. Considering a small refractive index increase, $\Delta \mathrm{n}$, and disregarding the square term, $\Delta n^{2}(x, y)$, it is possible rewrite equation (1) as[8]

$$
\Delta n(x, y)=\frac{\beta^{2}}{k_{o}^{2} 2 n_{B}}-\frac{n_{B}}{2}-\frac{\nabla_{\mathrm{T}}^{2} \sqrt{I(x, y)}}{k_{o}^{2} 2 n_{B} \sqrt{I(x, y)}}
$$

where $I(x, y)$ is the near field intensity measured experimentally and $\mathrm{n}_{\mathrm{B}}$ the refractive index of the surrounding medium, in this case the LiF bulk value at the selected wavelength. (ii) The estimated maximum $\Delta n_{\mathrm{H}}$ and $\Delta n_{\mathrm{V}}$ are used to simulate the NFP of an optical waveguide at the same wavelength of the light source used to obtain the experimental NFP. Due the unknown core shape of the waveguides, the NFP simulation considers a simplified rectangular core transverse section and TE polarization. The propagation constant is obtained from effective index method and the spatial field distribution from Marcatili's Method [1, 17]. The waveguide transverse section dimensions, $\mathrm{H}$ and $\mathrm{V}$, related to the horizontal and vertical directions, respectively, are adjusted to achieve the best fit between experimental and simulated NFP. The results consist of a set of transverse section dimensions as function of the RI estimate for the horizontal and vertical directions. (iii) Finally, in order to corroborate the estimate values of RI and core dimensions, the procedure is applied using NFP measured with light sources at other two wavelengths, $460 \mathrm{~nm}$ and $1550 \mathrm{~nm}$, as well at $635 \mathrm{~nm}$. The values of $\Delta n, H$ and $V$ are adjusted to achieve best fitted experimental and simulated NFP for the set of three different wavelengths. The final data consist of a set of $\Delta n$ values, each one related to a considered wavelength and just one couple of dimensions $H$ and $V$ for each waveguide. The code was implemented in MATLAB.

\section{B. Numerical Test on Optical Fibers}

The NFP simulation procedure is applied to the analysis of two commercial optical fibers (FSSN3224-SM @ 632.8 nm and COA-SMMF-SM @ $1550 \mathrm{~nm}$. The proposed approach was tested using the maximum $\Delta \mathrm{n}$ values estimated from the measured NFP for both optical fibers. The results were also compared with the step-index profile provided by the manufacturers. The NFP measurements were performed by using a semiconductor laser at $635 \mathrm{~nm}$ and a broad C-band source.

As an example, Fig. 2a shows the $\Delta n_{\mathrm{H}}$ estimated from the measured NFP together with that value provided by the manufacturer for the single mode fiber at $1550 \mathrm{~nm}$. Fig. $2 \mathrm{~b}$ shows the experimental NFP compared with those calculated by using the $\Delta n$ maximum values reported in Fig. 2a and the $\Delta n$ reported by the manufacturer. 

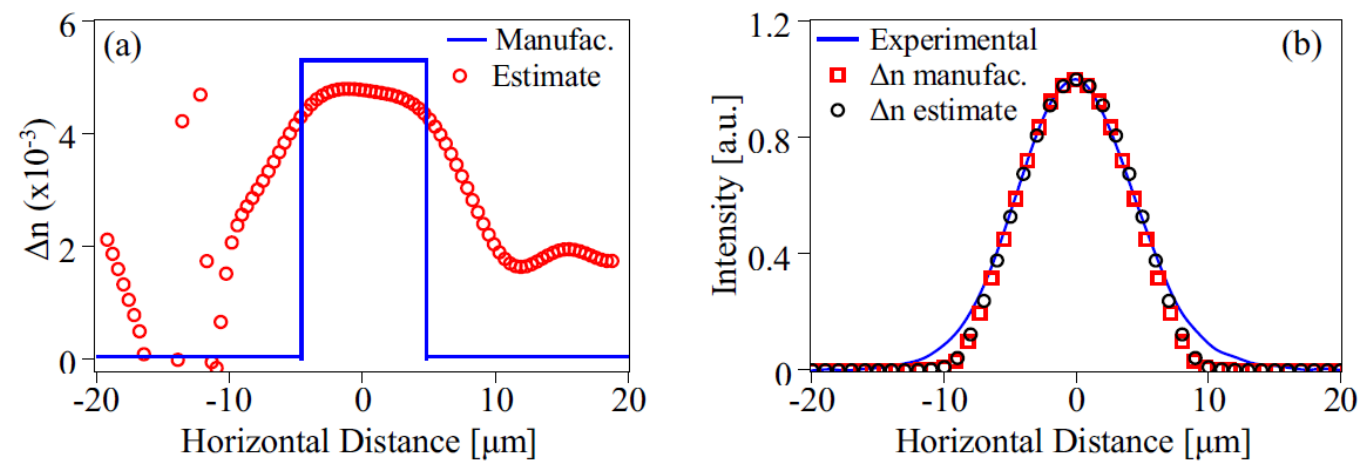

Fig. 2. (a) $\Delta$ n spatial profiles provided by the manufacturer and the estimated one derived from the NFP measured at $1550 \mathrm{~nm}$ and (b) the experimental and simulated NFP using the RI provided by manufacturer and the maximum value of the estimated $\Delta \mathrm{n}$ for the COA-SMMF-SM sample.

In Fig. $2 b$ the central region of the simulated intensity profile (from its maximum to about $35 \%$ of it) agrees well with the measured NFP for both wavelengths, the absolute mean errors are less than $1 \%$ and than $2 \%$ for the single mode optical fibers at $632.8 \mathrm{~nm}$ and $1550 \mathrm{~nm}$, respectively. The tails show differences attributed to the transverse core geometry, circular for the commercial optical fibers and rectangular in the simulations. Table I shows the values of the full width at half maximum of NFP as derived from measurements and from the simulations for the two optical fibers at both wavelengths, using the $\Delta n$ values provided by the manufacturer and those estimated by the NFP. In both case, the differences are consistent with the uncertainty of the used NFP measure system $(\sim 5 \%)$ and agree with the values provided by the optical fibers manufacturers: $4.0 \pm 0.5 \mu \mathrm{m}$ and $10.5 \pm 0.8 \mu \mathrm{m}$ for single mode optical fibers at $632.8 \mathrm{~nm}$ and $1550 \mathrm{~nm}$, respectively.

TABLE I. OPTICAL FIBERS MODAL FIELD DIAMETER, FWHM $[\mu \mathrm{m}]$, AT TWO WAVELENGTHS.

\begin{tabular}{ccccc}
\hline \multirow{2}{*}{ Optical Fiber } & Direction & Experimental & \multicolumn{2}{c}{ Calculated } \\
& & & $\Delta n$ Manufacturers & $\Delta n$ Estimated \\
\hline \multirow{2}{*}{ SM@632.8nm } & $H$ & 4.05 & 4.01 & 4.06 \\
& $V$ & 4.11 & 4.01 & 4.07 \\
\hline \multirow{2}{*}{ SM@1550nm } & $H$ & 10.45 & 10.16 & 10.37 \\
& $V$ & 10.56 & 10.14 & 10.34 \\
\hline
\end{tabular}

\section{Experimental Details}

We analyzed color-center optical waveguides recorded in a LiF crystal, which has a RI of 1.39599, 1.39123 and 1.38275 at the wavelengths $457.9 \mathrm{~nm}, 635 \mathrm{~nm}$ and $1550 \mathrm{~nm}$, respectively [18]. The crystal was directly irradiated with a focused femtosecond laser beam using a $20 \mathrm{X}$ microscope objective. The laser has $1 \mathrm{kHz}$ repetition rate and emission band centered at $800 \mathrm{~nm}$. The optical waveguides were recorded at depths of $\sim 160 \mu \mathrm{m}$ below the polished crystal top surface. The NFP was measured launching different light sources in the waveguides by a $10 \mathrm{X}$ microscope objective. At the end of the waveguide a similar objective collimated the emerging light into Thorlabs Beam Profiler 
(models BP104UV and BP104IR, depending on the used wavelength). The microscope objectives and the LiF crystal were assembled on translation stages Newport ULTRAlign 561 series to achieve the optical alignment. The LiF crystal is hard and it is difficult to properly polish or cleave its side facets. This implies that mechanical damages generated by the focalized laser beam on the entrance and exit sides during the waveguides recording process could not be fully eliminated. Methods that require high quality of the NFP measure can have limitations under such conditions $[8,9]$.

\section{RESULTS AND DISCUSSION}

Using the described approach the core dimensions and the corresponding $\Delta \mathrm{n}$ are adjusted to match the experimental NFP at each of the used wavelengths. Table II shows the recording parameters of the LiF waveguides (laser energy and sample translation velocity), the calculated values of the core dimensions and $\Delta n$ increase, from the best fitted simulated NFP.

TABLE II. RECORDING PARAMETERS, CORE DIMENSIONS AND REFRACTIVE INDEX CHANGE VALUES ESTIMATED AT THREE WAVELENGTHS FOR SEVERAL LIF WAVEGUIDES.

\begin{tabular}{cccccccc}
\hline & \multicolumn{6}{c}{ Core } & \multicolumn{3}{c}{$\Delta n\left(\mathrm{x} 10^{-4}\right)$} & \\
Guide\# & $\mathrm{E}$ & $\mathrm{v}$ & \multicolumn{2}{c}{ dimensions } & & \\
& {$[\mu \mathrm{J}]$} & {$[\mu \mathrm{m} / \mathrm{s}]$} & $\begin{array}{c}H \\
{[\mu \mathrm{m}]}\end{array}$ & $\begin{array}{c}V \\
{[\mu \mathrm{m}]}\end{array}$ & $460 \mathrm{~nm}$ & $635 \mathrm{~nm}$ & $1550 \mathrm{~nm}$ \\
\hline 4 & 2 & 241 & 8.1 & 10.9 & 8 & 5.9 & 3.0 \\
5 & 5 & 241 & 8.2 & 10.0 & 9 & 3.8 & 2.2 \\
6 & 5 & 191 & 8.3 & 9.6 & 8 & 4.0 & 2.3 \\
7 & 2 & 191 & 8.3 & 10.2 & 10 & 6.1 & 2.8 \\
\hline
\end{tabular}

Fig. 3(a, c) shows the experimental NFP as measured at $635 \mathrm{~nm}$ and after numerical processing with a $1 \mathrm{~Hz}$ low pass filter, $I$ and $I_{f}$, respectively, for the horizontal (Fig. 3a) and vertical (Fig. 3c) directions. From these plots it is possible to see that the filtering process influences only the noise on the $\Delta \mathrm{n}$ estimate, without distorting its main features, as shown in Fig. 3(b) and (d). According with these values, the experimental and simulated NFP for the waveguide \#4 on horizontal and vertical directions are shown in Fig. 4(a) and (b), respectively. The difference between the simulated and measured NFP intensity profiles outside the central region can be attributed to several factors, including scattering of light at damage regions caused by laser ablation at the waveguide exits, multi modal propagation, leaky modes and interference patterns generated by reflections. Disregarding these side regions, the measured intensity profiles agree well with the simulated NFP.

Table III summarizes the values of the full width at half maximum of the experimental and simulated NFP corresponding to the dimensions and refractive index differences given in Table II for several wavelengths. 

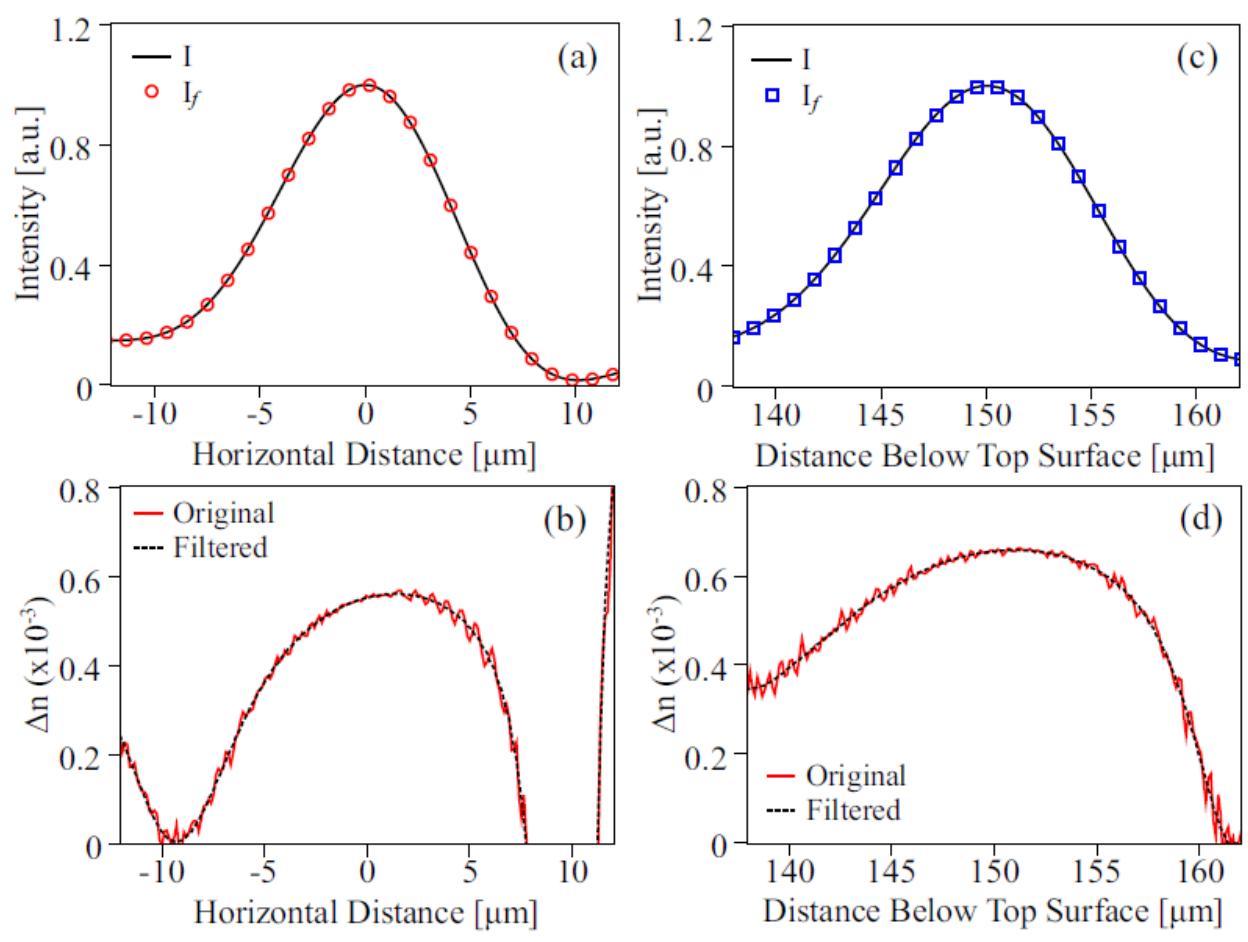

Fig. 3. Measured, $I$, and filtered, $I_{f}$, experimental NFP (a,c) and $\Delta$ n estimate (b,d) on horizontal (a-b) and vertical (c-d) directions of waveguide \#4 at $635 \mathrm{~nm}$.
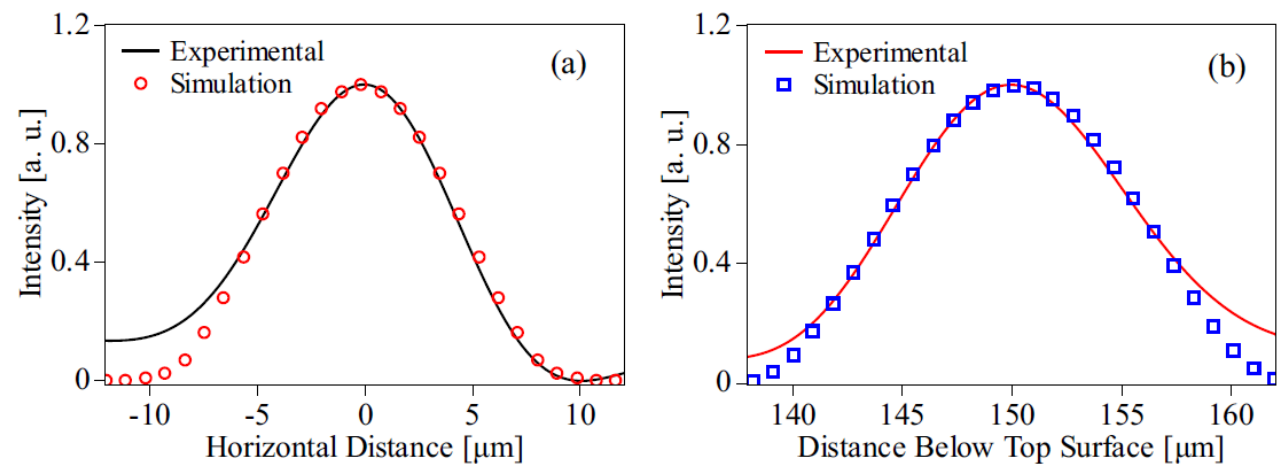

Fig. 4. Experimental and simulated NFP of waveguide \#4 at $635 \mathrm{~nm}$, for (a) horizontal and (b) vertical directions.

TABLE III EXPERIMENTAL AND CALCULATED FWHM OF SEVERAL LIF WAVEGUIDES AT THREE WAVELENGTHS.

\begin{tabular}{cccccccc}
\hline \multirow{2}{*}{ Guide\# } & \multicolumn{7}{c}{ FWHM [ $[\mu \mathrm{m}]$ at wavelengths } \\
& & & \multicolumn{2}{c}{$635 \mathrm{~nm}$} & \multicolumn{2}{c}{$1550 \mathrm{~nm}$} \\
& & $H$ & $V$ & $H$ & $V$ & $H$ & $V$ \\
\hline \multirow{2}{*}{4} & Exp. & 9.3 & 12.1 & 9.7 & 12.6 & 16.8 & 15.5 \\
& Sim. & 9.0 & 11.9 & 9.8 & 12.5 & 14.5 & 17.0 \\
\hline \multirow{2}{*}{5} & Exp. & 9.5 & 10.1 & 10.6 & 12.8 & 18.7 & 14.9 \\
& Sim. & 9.1 & 10.9 & 10.3 & 12.1 & 16.4 & 17.9 \\
\hline \multirow{2}{*}{6} & Exp. & 9.5 & 9.8 & 10.6 & 12.5 & 17.7 & 17.0 \\
& Sim. & 9.2 & 10.5 & 10.4 & 12.0 & 15.7 & 17.1 \\
\hline \multirow{2}{*}{7} & Exp. & 9.6 & 11.3 & 9.8 & 12.0 & 16.7 & 16.7 \\
& Sim. & 9.2 & 11.1 & 9.9 & 11.8 & 15.0 & 16.6 \\
\hline
\end{tabular}


At $460 \mathrm{~nm}$ and $635 \mathrm{~nm}$ the differences in the values of core dimensions directly derived from NFP and those calculated on the basis of the $\Delta n$ values of Table II are less than $8 \%$. The greater mismatch at $1550 \mathrm{~nm}$ can be ascribed to effects of the broadband light source used to measure the profile, with respect to the single line wavelength used for the simulation. In this case, the experimental NFP measures consist of a modal profile composition as a function of the wavelength.

\section{CONCLUSIONS}

The results obtained with the proposed approach, together with those obtained in the numerical test performed on commercial optical fibers, show the possibility to estimate the core dimensions and the average increase of the refractive index of $\mathrm{LiF}$ optical waveguides without the need of knowing details about the core shape and $\Delta n$ profile. Assuming a simplified rectangular transversal section, the $\Delta \mathrm{n}$ increase and the transversal dimensions were estimated by a recursive procedure used to compare the experimental and simulated NFP of the waveguides at several wavelengths. The recursive procedure permits to estimate these parameters from measured data with simple numerical treatments.

The estimated $\Delta \mathrm{n}$ values obtained for optical waveguides recorded by fs laser pulses in LiF are in order of $(2-10) \times 10^{-4}$, at wavelengths in the visible to near-infrared spectral range and the best fitted core dimensions are $\sim(8 \mathrm{x} 10) \mu \mathrm{m}^{2}$. The obtained results also support the possibility of recording low index contrast buried optical waveguides in lithium fluoride crystal by femtosecond laser irradiation, having single to few modes of propagation.

\section{ACKNOWLEDGMENT}

The authors thank Dr. Vladimir Kalinov for providing the used blank lithium fluoride crystals; financial support from CNPq, CAPES, FINEP and Fundação Araucária (Brazilian Agencies) is also highlighted. Dr. Kalinowski acknowledges support from ICTP (Italy), through its Senior Associate Programme, for visiting periods at ENEA C.R. Frascati.

\section{REFERENCES}

[1] K. OKAMOTO, Fundamentals of Optical Waveguides. 2 ed. Amsterdam: Elsevier Inc., 2006.

[2] G. E. AIZENBERG, P. L. SWART and B. M. LACQUET, "A Digital Signal-Processing Analysis Technique for The Infrared Reflectivity Characterization of Ion-Implanted Silicon” Journal of Electronic Materials, vol. 21, pp. 10331040, 1992

[3] T. OKOSHI, Optical Fibers, First, Ed. Academic Press Inc., 1982.

[4] C. J. ALLEYNE, et al. "Numerical method for high accuracy index of refraction estimation for spectro-angular surface plasmon resonance systems” Optics Express, vol. 16, pp. 19493-19503, 2008.

[5] X. H. LIU, et al. "Optical properties of a single mode planar waveguide in Nd:YVO4 fabricated by multienergy He ion implantation” Applied Optics, vol. 50, pp. 3865-3870, 2011. 
[6] P. J. CHANDLER, F. L. LAMA, "A new approach to the determination of planar waveguide profiles by means of a non stationary mode index calculation," Optica Acta, vol. 33, pp. 127-143, 1986.

[7] A. CHIASERA, et al. "CO2 Laser irradiation of GeO2 planar waveguide fabricated by rf-sputtering" Optical Materials Express, vol. 3, pp. 1561-1570, 2013.

[8] M. L. BIBRA, A. ROBERTS, "Refractive Index Reconstruction of Graded-Index Buried Channel Waveguides from Their Mode Intensities," Journal of Lightwave Technology, vol. 15, pp. 1695-1699, 1997.

[9] S. Y. XU, et al "Refractive Index Profile in Photorefractive-Damage-Resistant Near-Stoichiometric Ti:Mg:Er:LiNbO3 Strip Waveguide” IEEE Photonics Journal, vol. 4, pp. 1823 - 1830, 2012.

[10] W. S. TSAI, S. C. PIAO, P. K. WEI, "Refractive index measurement of optical waveguides using modified end-fire coupling method," Optics Letters, vol. 36, pp. 2008-2010, 2011.

[11] K. M. DAVIS, et al. "Writing waveguides in glass with a femtosecond laser.," Optics Letters, vol. 21, no. 21, pp. 17291731, 1996

[12] H. ZHANG, S. M. EATON, and P. R. HERMAN, "Single-step writing of Bragg grating waveguides in fused silica with an externally modulated femtosecond fiber laser," Optics Letters, vol. 32, no. 17, pp. 2559-2561, 2007.

[13] R. OSELLAME, et al. "Optical waveguide writing with a diode-pumped femtosecond oscillator," Optics Letters, vol. 29, pp. 1900-1903, 2004.

[14] G. BROWN, et al. "Ultrafast laser inscription of Bragg-grating waveguides using the multiscan technique," Optics Letters, vol. 37, pp. 491-493, 2012.

[15] I. CHIAMENTI, et al. “ Broadband Optical Active Waveguides Written by Femtosecond Laser Pulses in Lithium Fluoride” Chinese Physics Letters, vol. 31, pp. 014201-014201-4, 2014.

[16] I. CHIAMENTI, et al. "Optical characterization of femtosecond laser induced active channel waveguides in lithium fluoride crystals” Journal of Applied Physics, vol. 115, pp. 023108 - 023108-7, 2014.

[17] M. ADAMS, Introduction to Optical Waveguide. John Wiley I\& Sons Ltd., 1981.

[18] E. D. PALIK, Handbook of Optical Constants of Solids. Academic Press, New York, 1985. 